\title{
Manejo da cárie dentária e comportamento infantil durante a pandemia de COVID-19: relato de caso
}

\author{
Monique Gonzaga BARBOSA ${ }^{1}$; Nara Rúbia da SILVA'; Sejane \\ Sousa Barros OLIVEIRA'; Patrícia CORRÊA-FARIA ${ }^{2}$
}

1 - Graduanda em Odontologia, Faculdade Sul-Americana, Goiânia-GO, Brasil; 2 - Professora do Departamento de Odontologia, Faculdade Sul-Americana, Goiânia-GO, Brasil.

\section{Resumo}

O objetivo deste artigo é relatar um caso clínico de manejo da cárie dentária e do comportamento infantil no cenário da pandemia de COVID-19. Um menino, sete anos, se queixou de cárie e ausência de colaboração durante atendimentos anteriores devido ao medo/ansiedade. No exame clínico foram observadas lesões cariosas cavitadas (dentes $51,55,61,63,64,73,74,83,85$ ) e/ ou destruição coronária (54, 75 e 84). Propôs-se a restauração das lesões sem envolvimento pulpar usando-se a técnica de tratamento restaurador atraumático para preservar a estrutura dentária e reduzir a produção de aerossóis; e exodontia nos casos de extensa destruição coronária. Para reduzir o medo/ansiedade infantil, foram usadas técnicas básicas de manejo do comportamento infantil. Ansiedade e comportamento foram avaliados usando-se escalas para permitir a avaliação da eficiência das técnicas de manejo. As medidas de segurança foram explicadas à criança (uso de protetor facial, distanciamento na sala de espera, presença de apenas um acompanhante). Realizou-se remoção seletiva do tecido cariado e restauração com cimento de ionômero de vidro nos dentes $63,64,73,74,83,85$. Nestes procedimentos, a criança teve comportamento colaborador. Os dentes 75 e 84 foram extraídos sob estabilização protetora devido ao comportamento não colaborador. Ao final, a criança foi encaminhada para acompanhamento ortodôntico. Observou-se redução da ansiedade, melhora do comportamento e controle da doença cárie. As técnicas minimamente invasivas são uma opção para o manejo da cárie dentária. Para reduzir o medo/ansiedade infantil recomenda-se o uso de técnicas básicas de manejo de comportamento.

PALAVRAS-CHAVE: Infecções por coronavirus; Cárie dentária; Comportamento infantil.

Copyright @ 2021 Revista Odontológica do Brasil Central Esta obra está licenciada com uma licença Atribuição-NãoComercialCompartilhalgual 4.0 Internacional (CC BY-NC-SA 4.0)

Recebido: 20/01/21 Aceito: $15 / 03 / 21$ Publicado: 05/04/21

\section{AUTOR PARA CORRESPONDÊNCIA}

Patrícia Corrêa-Faria

Faculdade Sul-Americana - BR-153, Km 502, Jardim da Luz - Goiânia/GO, Brasil, CEP. 74.850-370

E-mail: patriciafaria.faria09@gmail.com 


\section{Introdução}

A pandemia de COVID-19 mudou o cenário do atendimento odontopediátrico. Para evitar a contaminação da equipe odontológica, do paciente e da sua família/cuidador, medidas de biossegurança mais rígidas foram propostas devendo ser seguidas desde o momento de acolhida da criança até o término do atendimento. Medidas para reduzir a produção de aerossóis durante os procedimentos odontológicos também foram instituídas e impactaram na decisão de tratamento da cárie dentária ${ }^{1}$.

Publicações nacionais e internacionais recomendam que o manejo da cárie dentária seja realizado usando procedimentos minimamente invasivos ${ }^{1-4}$. Essa abordagem envolve procedimentos não invasivos, como o controle do biofilme, da dieta e da mineralização das estruturas dentárias; e outros micro invasivos e invasivos, como a aplicação de selantes e as restaurações, respectivamente, que têm o objetivo de preservar, ao máximo a estrutura dentária e gerar condições favoráveis para a remineralização dos tecidos ${ }^{5}$. Além do aspecto biológico de preservação da estrutura dentária, os procedimentos minimamente invasivos são reconhecidos como "amigáveis" por auxiliarem na redução da ansiedade e no melhor manejo do comportamento infantil'.

As técnicas para manejo do comportamento infantil são um tema frequente nas recomendações para o tratamento odontopediátrico durante a pandemia de COVID-197,8. O uso de técnicas básicas é encorajado para reduzir o medo e/ou a ansiedade das crianças ao lidarem com as mudanças no ambiente odontológico e na paramentação da equipe. Para isso, recomenda-se que a criança receba explicações sobre a necessidade de distanciamento dos demais pacientes na sala de espera e sobre os equipamentos de proteção individual usados pela equipe e se sinta acolhida e protegida no ambiente odontológico ${ }^{9,10}$. A adoção das recomendações de biossegurança, manejo da cárie e do comportamento pode ser um desafio para os dentistas e alunos de graduação. 
Diante disso, o objetivo desse estudo é relatar um caso de atendimento odontológico de uma criança com cárie dentária durante a pandemia de COVID-19, enfatizando-se o uso de procedimentos minimamente invasivos e de técnicas para o manejo do comportamento.

\section{Relato do caso}

Este relato de caso foi descrito de acordo com as diretrizes CARE ${ }^{11}$. A responsável legal do paciente assinou um termo de consentimento livre e esclarecido autorizando a realização do tratamento e a divulgação de informações e imagens referentes ao caso clínico.

Um menino de sete anos de idade compareceu na clínica de odontopediatria da Faculdade Sul-Americana (FASAM), Goiânia-GO, com queixa de cárie e dor provocada por alimentos doces. Não foi observada alteração no estado de saúde da criança e, na anamnese foi relatado comportamento não colaborador em consultas odontológicas anteriores. No exame intraoral observaram-se lesões cariosas cavitadas e ativas nos dentes 55, 54 (remanescente radicular), 51, 61, 63, 64, 73, 74, 75 (remanescente radicular), 83, 84, 85 (Figura 1). Radiograficamente, confirmou-se que a lesão cariosa se estendia à polpa dos dentes 55, 84 (Figura 2).

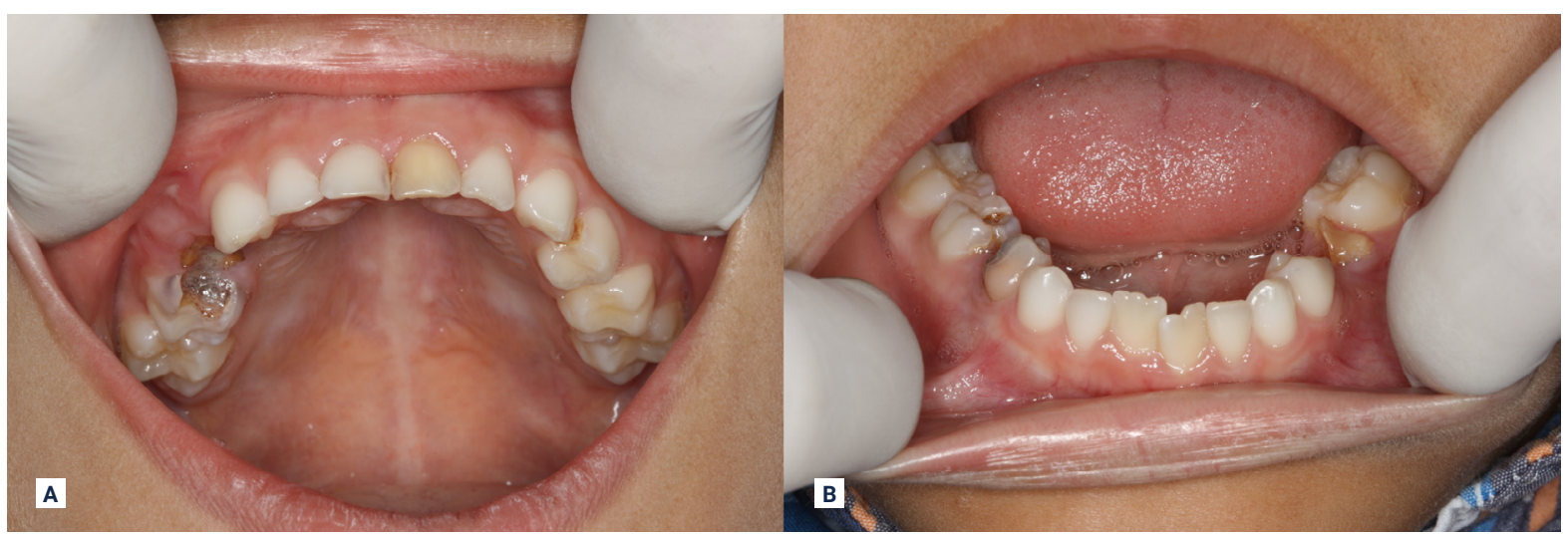

FIGURA 1 - Aspecto clínico inicial: (A) arcada superior: lesões cariosas cavitadas nos dentes 51, 55, 61, 63 e 64; remanescentes radiculares do dente 54; (B) arcada inferior: lesões cariosas cavitadas nos dentes $74,75,84$ e 85 
Foram realizadas tomadas radiográficas intrabucais para verificar a extensão das lesões cariosas e extraoral para acompanhamento do desenvolvimento da dentição. O dente 51 apresentava descoloração devido a um traumatismo. Diante disso, propôs-se a restauração das lesões cavitadas em que não havia comprometimento do tecido pulpar e exodontia dos remanescentes radiculares (54 e 75) e dos dentes 55 e 84 . Optou-se por não restaurar as cavidades dos incisivos centrais superiores devido ao avançado grau de esfoliação.

Previamente a cada consulta, o estado de saúde da criança e da acompanhante foi verificado por meio de contato telefônico e, na sala de espera, a temperatura corporal foi medida. A acompanhante foi orientada a permanecer com máscara durante todo o tempo. Adicionalmente, na primeira sessão, a equipe odontológica explicou à criança, usando termos simples, a necessidade de a operadora e auxiliar usarem o protetor facial. Para auxiliar na aproximação com a criança e formação de vínculo, uma fotografia da operadora e auxiliar permaneceu à vista da criança ${ }^{12}$ (Figura 3).

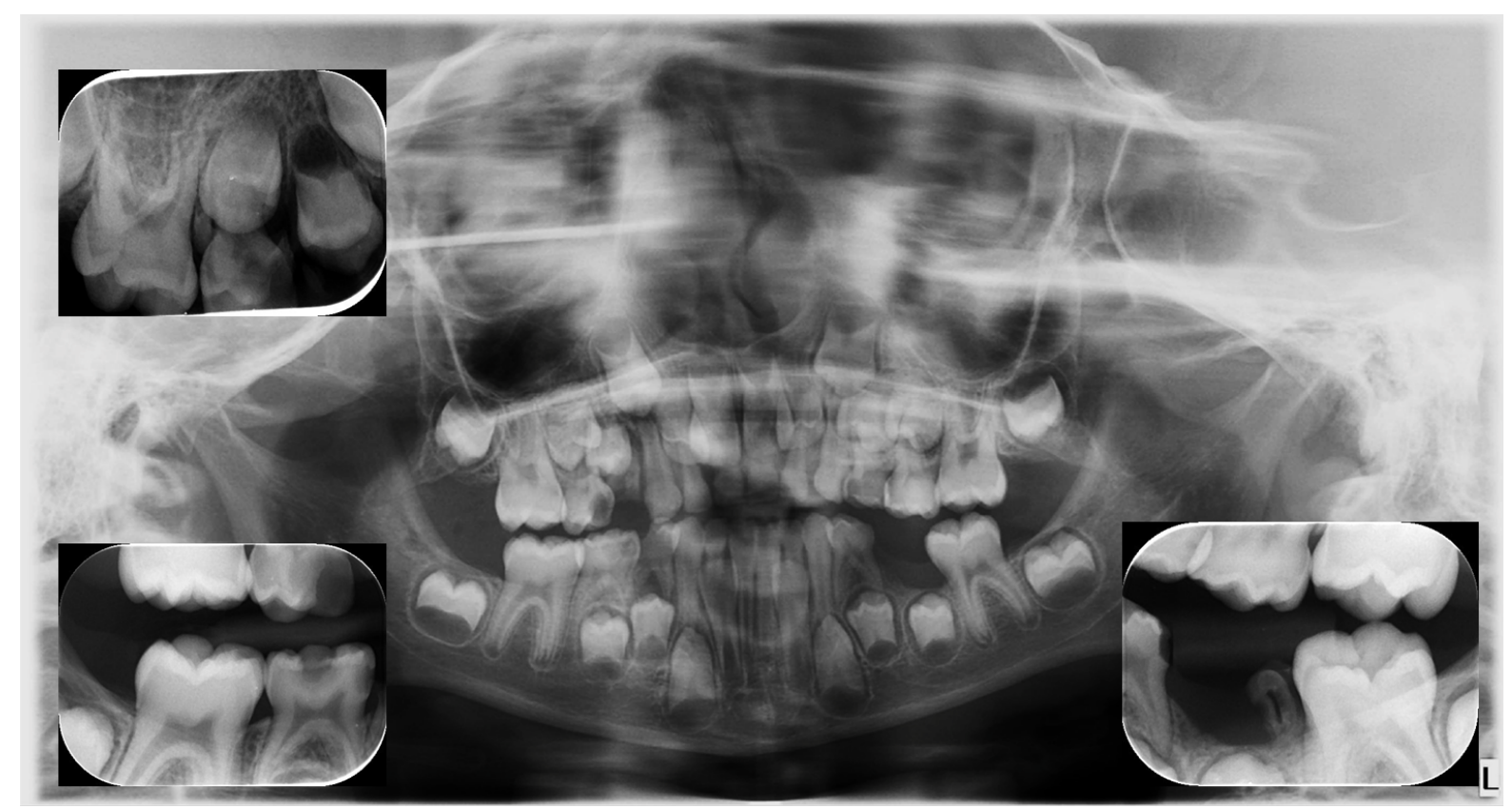

FIGURA 2 - Aspecto radiográfico inicial e durante o tratamento 
O tratamento das lesões cavitadas sem comprometimento pulpar foi realizado usando-se a técnica de tratamento restaurador atraumático. Inicialmente, foi realizada profilaxia utilizando-se pedra pomes e escova de Robinson. Em seguida, sob isolamento relativo, o tecido cariado observado nas paredes circundantes foi completamente removido e, realizou-se remoção seletiva da dentina cariada localizada na parede de fundo das cavidades. As cavidades foram condicionadas com ácido poliacrílico, lavadas, secas e preenchidas com cimento de ionômero de vidro restaurador (Maxxion $\mathrm{R}, \mathrm{FGM}^{\circledR}$, Joinville - SC, Brasil). O material restaurador foi comprimido na cavidade com auxílio de dedo enluvado e vaselinado. Após a presa inicial, o excesso de material restaurador foi removido, checados os contatos oclusais e realizada proteção por meio da reaplicação de uma fina camada de vaselina (Vasemax, Farmax $^{\circledR}$, Minas Gerais). Quando a cavidade se localizava ou se estendia à superfície proximal, a restauração foi realizada após a adaptação de matriz de aço e cunha de madeira. A anestesia local e isolamento absoluto foram dispensados, conforme permitido na técnica usada ${ }^{13}$. Ao final das restaurações, realizadas em quatro sessões, o comportamento da criança foi classificado pela equipe odontológica como definitivamente positivo ${ }^{14}$.

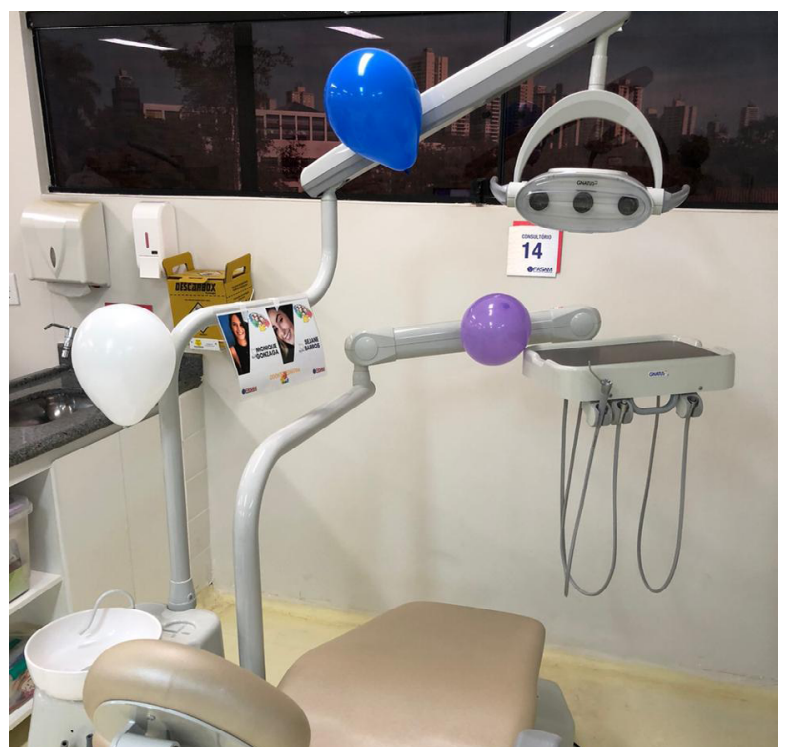

FIGURA 3 • Equipo odontológico com balões e fotografia da equipe odontológica 
As exodontias foram realizadas em quatro sessões intercaladas com aquelas destinadas aos procedimentos restauradores. Em cada sessão foi realizada apenas uma extração. Os procedimentos foram realizados sob anestesia local (lidocaína a $2 \%$ com adrenalina 1:100.000, Alphacaine, DFL ${ }^{\circledR}$, Rio de Janeiro) e, quando necessário, estabilização protetora. Quando a estabilização protetora foi usada, os membros da criança foram contidos pela responsável legal que foi previamente esclarecida sob a técnica e consentiu com seu uso, por escrito. Ao final dessas sessões, o comportamento infantil foi classificado como positivo (duas sessões) e definitivamente negativo (demais sessões) ${ }^{14}$. Na figura 4 observam-se as restaurações e adequada cicatrização das regiões em que foram realizadas as exodontias.

A ansiedade infantil foi autorrelatada antes de cada sessão de tratamento. Para isso, cinco faces representando, progressivamente, diferentes níveis de ansiedade foram apresentadas à criança e solicitado que apontasse o rosto correspondente a como ela estava se sentindo naquele momento (Facial Image Scale - FIS) ${ }^{15}$ (Figura 5).

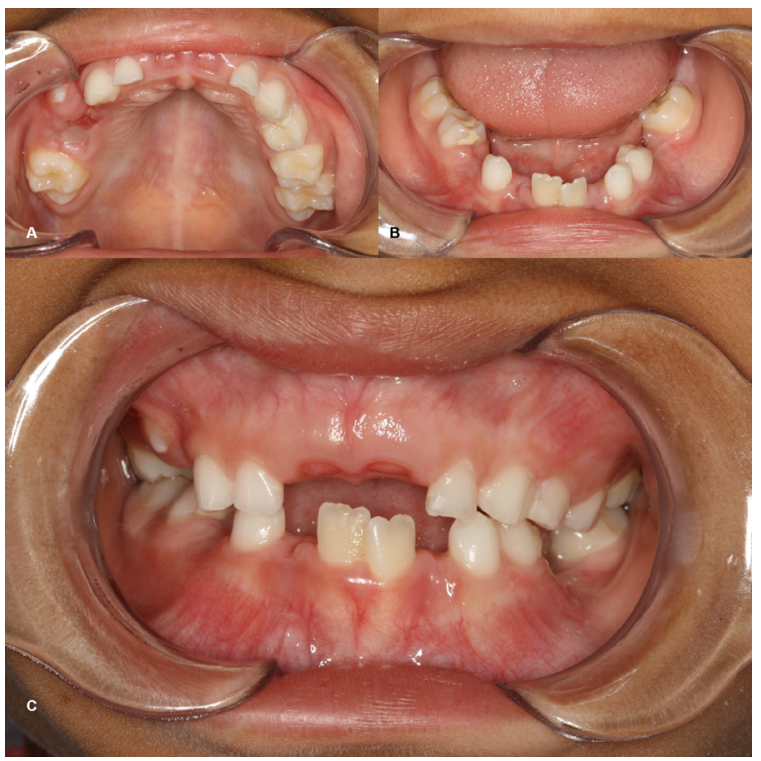

FIGURA 4 - Aspecto clínico final: A) arcada superior: restauração na face distal do dente 63; cicatrização da região correspondente aos dentes 64 e 65; B) arcada inferior: restaurações nos dentes 74 (distal) e 85 (ocluso-mesial); cicatrização da região dos dentes 75 e 84; C) aspecto final das arcadas em oclusão

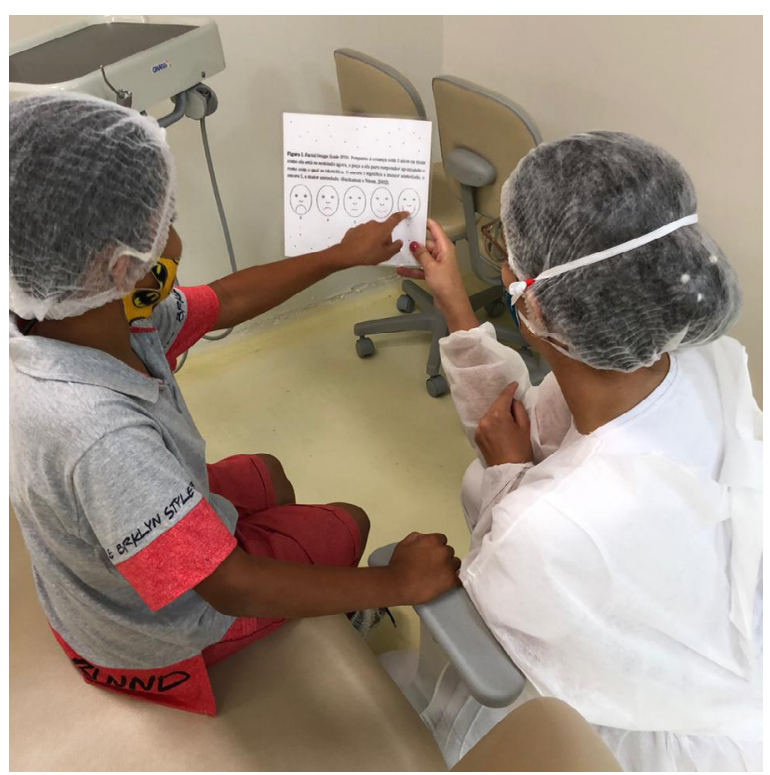

FIGURA 5 • Aplicação da Facial Image Scale para avaliação da ansiedade infantil previamente às sessões de tratamento 
Observou-se que, na maioria das avaliações, a criança não se sentiu ansiosa ou estava pouco ansiosa (escore 1 a 3 da FIS). Nas sessões em que a criança relatou estar ansiosa (escores 2 e 3) houve comportamento não colaborador (escores 1 e 2 da escala de Frank $1^{14}$ ) durante os procedimentos.

Para reduzir o medo/ansiedade e estimular um comportamento colaborador da criança, foram usadas técnicas básicas de manejo de comportamento ${ }^{16}$. Em todas as sessões, foram aplicadas as técnicas falar-mostrar-fazer, distração e, quando apropriado, o reforço positivo.

Ao final do tratamento odontopediátrico, a criança foi encaminhada para acompanhamento do desenvolvimento da oclusão e instalação de um aparelho mantenedor de espaço. A criança e a responsável relataram se sentir satisfeitos com o tratamento e observou-se adesão às recomendações para manutenção da saúde bucal. A responsável legal relatou perceber melhorias no comportamento da criança e redução da ansiedade prévia às consultas odontológicas.

\section{Discussão}

Neste artigo, foi relatado um caso de manejo de cárie dentária e comportamento infantil seguindo-se as recomendações atuais para atendimento odontopediátrico durante a pandemia de COVID-19. Medidas de biossegurança foram rigorosamente seguidas para reduzir o risco de contaminação cruzada e a fim de proporcionar um atendimento mais confortável para a criança, foram aplicadas técnicas de manejo de comportamento e procedimentos minimamente invasivos, quando apropriado.

Durante a pandemia de COVID-19 foram propostas mudanças no atendimento odontológico para reduzir o risco de contaminação da equipe e pacientes. Essas mudanças envolveram a suspensão momentânea de procedimentos eletivos ${ }^{17,18}$, preferência por procedimentos minimamente invasivos para manejo da 
cárie dentária visando a redução ou eliminação da geração de aerossol $^{1,2,7,12}$, estímulo às atividades educativas e de promoção de saúde e recomendação, ainda mais rigorosa, do uso de equipamentos de proteção individual ${ }^{4,19}$. A adequação a essas recomendações é um desafio na prática odontológica, tanto nos consultórios quanto nas clínicas das instituições de ensino superior.

Neste estudo, foi descrito o caso de uma criança com extensa necessidade de tratamento odontológico, possivelmente agravada pela dificuldade de acesso ao serviço de saúde durante a pandemia. Em uma avaliação realizada no período de março 2019 a maio de 2020 se observou redução de 66\% no número de tratamentos pediátricos realizados no Sistema Único de Saúde $\left(\right.$ SUS ${ }^{20}$. Esse dado traz um alerta aos profissionais de saúde que devem estar preparados para lidar com a piora da situação bucal de muitos pacientes infantis.

Diante da extensa necessidade de tratamento odontológico da criança e da preocupação em prestar atendimento com segurança tanto para a equipe quanto para o paciente e seu cuidador propôs-se o manejo das lesões cariosas usando-se procedimentos minimamente invasivos, quando possível. O tratamento restaurador atraumático (TRA) foi o procedimento de escolha para manejo das lesões cariosas ativas em que não havia comprometimento do tecido pulpar. Nesta técnica, o tecido cariado é seletivamente removido usando instrumentos cortantes manuais e a cavidade é selada com material restaurador adesivo, como o cimento de ionômero de vidro ${ }^{13}$. O TRA permite uma abordagem conservadora, biológica e eficaz das lesões de cárie e, no contexto da pandemia, se destaca pelo menor risco de geração de aerossóis já que dispensa o uso de alta rotação ${ }^{1}$.

O TRA, somado às técnicas de manejo de comportamento, teve um papel importante na redução do medo/ansiedade infantil. No caso descrito, havia história de ausência de colaboração em atendimentos anteriores e ansiedade frente aos procedimentos. Essas queixas foram minimizadas ou pouco percebidas quando 
a criança foi submetida ao procedimento restaurador com o TRA. O uso de brocas e de anestesia são fatores reconhecidos como associados à ansiedade odontológica. A dispensa do seu uso no TRA parece contribuir para a minimização da ansieda$\mathrm{de}^{21} \mathrm{e}$, consequente, melhoria no comportamento infantil durante $\mathrm{o}$ atendimento.

Para reduzir o medo/ansiedade infantil foram aplicadas técnicas de manejo do comportamento ${ }^{16}$. As técnicas foram aplicadas desde a acolhida do paciente/cuidador até a realização dos procedimentos odontológicos. Diferentemente do momento pré-pandemia, na acolhida da criança, a equipe odontológica já está paramentada. A paramentação (protetor facial e máscara) pode causar estranhamento na criança e dificultar, em um primeiro momento, a aproximação e vínculo com a equipe. Para minimizar essa dificuldade, a equipe explicou à criança, de forma lúdica, a necessidade da paramentação e usou uma fotografia para se apresentar sem os equipamentos ${ }^{12}$. Percebeu-se que essas medidas foram eficientes e bem recebidas pela criança e seu cuidador.

Técnicas básicas como o falar-mostrar-fazer e a distração foram usadas em todas as sessões de tratamento para estabelecer uma interação contínua entre paciente, cuidador e equipe odontológica, aliviar o medo/ansiedade e promover uma atitude positiva em relação ao tratamento odontológico e saúde bucal ${ }^{16}$. Essas técnicas foram eficientes na redução do medo/ansiedade especialmente durante os procedimentos restauradores e bem aceitas pela díade criança/cuidador ${ }^{22}$.

Durante as exodontias, as técnicas básicas não foram suficientes e optou-se por conciliá-las com a estabilização protetora. Esta técnica tem uso restrito na odontopediatria e está indicada em contextos específicos como a mudança brusca do comportamento da criança de colaborador para não colaborador durante o procedimento e situações de urgência odontológica ${ }^{16}$. Ainda há poucas evidências sobre os efeitos do uso da estabilização 
protetora no desenvolvimento psicológico infantil. Assim, se enfatiza que a estabilização protetora deve ser recomendada após se avaliar, com cautela, os benefícios e riscos para a criança e a possibilidade de usar estratégias alternativas ${ }^{23}$ como o adiamento do procedimento ou o atendimento sob sedação ou anestesia geral ${ }^{16}$. No presente caso, o risco de adiar o procedimento era superior ao benefício e serviços que oferecem tratamento sob técnicas farmacológicas de manejo não são facilmente disponíveis.

No atendimento odontopediátrico durante a pandemia devem ser observadas as recomendações para reduzir o risco de contaminação do paciente, cuidador e equipe odontológica. Deve-se também proporcionar um atendimento confortável, com redução do medo/ansiedade e decidir por terapias que sejam eficazes na manutenção/recuperação da saúde bucal.

\section{Conclusão}

O tratamento odontopediátrico durante a pandemia deve proporcionar segurança e conforto ao paciente. A equipe odontológica deve estar atenta às medidas de biossegurança recomendadas para o contexto da pandemia de COVID-19, às técnicas que podem ser aplicadas para reduzir o medo/ansiedade e àquelas eficazes para o manejo da cárie dentária. Neste caso clínico, a interação entre a equipe, paciente e cuidador; e o uso de procedimentos minimamente invasivos permitiram a resolução das necessidades de tratamento da criança de modo seguro e eficiente.

\section{Referências}

1 - Asociación Latinoamericana de Odontopediatría. Tratamiento de caries en época de COVID-19: Protocolos clínicos para el control de generación de aerosoles. Rev Latinoam Odontop. 2020; 10(2): 1-28.

2 - BaniHani A, Gardener C, Raggio DP, Santamaría RM, Albadri S. Could COVID-19 change the way we manage caries in primary teeth? Current implications on Paediatric Dentistry. Int J Paediatr Dent. 2020; 30(5): 523-525. 
3 - Mallineni SK, Innes NP, Raggio DP, Araujo MP, Roberstson MD, Jayaraman J. Cononavirus disease (COVID-19): characteristics in children and considerations for dentists providing their care. Int J Paediatr Dent. 2020; 30(3): 245-250.

4 - Shah S. COVID-19 and paediatric dentistry: traversing the challenges. A narrative review. Ann Med Surg (Lond). 2020; 58: 22-33.

5 - Innes NP, Manton DJ. Minimum intervention children's dentisty: the starting point for a lifetime of oral health. Br Dent J. 2017; 223(3): 205-213.

6- Roberts JF, Curzon MEJ, Martens LC. Review: behaviour management techniques in paediatric dentistry. Eur Arch Paediatr Dent. 2010; 11(4): 166-174.

7 - Jayaraman J, Dhar V, Moorani Z, Donly K, Tinanoff N, Mitchell S, Wright T. Impact of COVID-19 on pediatric dental practice in the United States. Pediatr Dent. 2020; 42(3): 180-183.

8 - Ilyas N, Agel M, Mitchell J, Sood S. COVID-19 pandemic: the first wave: na audit and guidance for paediatric dentistry. Br Dent J. 2020; 228(12): 927-931.

9- Acharya S, Singh B, Godhi B, Pandey S. How to deal and learn from the threat of COVID-19 in paediatric dentistry. Eur J Paediatr Dent. 2020; 21(3): 173-175.

10 - Amorim LM, Maske TT, Ferreira SH, Santos RB, Feldens CA, Kramer PF. New post-COVID-19 biosafety protocols in pediatric dentistry. Pesqui Bras Odontopediatria Clín Integr. 2020; 20(supp1): e0117.

11 - Gagnier JJ, Kienle G, Altman DG, Moher D, Sox H, Riley D, CARE Group. The CARE Guidelines: consensus-based clinical case reporting guidelines development. Glob Adv Health Med. 2013; 2(5): 38-43.

12 - Al-Halabi M, Salami A, Alnuaimi E, Kowash M, Hussein I. Assessment of paediatric dental guidelines and caries management alternatives in the post COVID-19 period. A critical review and clinical recommendations. Eur Arch Paediatr Dent. 2020; 21(5): 543-556.

13 - Leal S, Bonifacio C, Raggio D, Frencken J. Atraumatic restorative treatment: restorative component. Monogr Oral Sci. 2018; 27: 92-102.

14 - Frankl S, Shiere F, Fogels H. Should the parent remain with the child in the dental operatory. J Dent Child. 1962; 29: 150-163.

15 - Buchanan H, Niven N. Validation of a facial image scale to assess child dental anxiety. Int J Paediatr Dent. 2002; 12(1): 47-52.

16 - American Academy of Pediatric Dentistry. Behavior guidance for the pediatric dental patient. The reference manual of pediatric dentistry. Chicago, Ill.: American Academy of Pediatric Dentistry; 2020. p. 292-310.

17 - Peng X, Xu X, Li Y, Cheng L, Zhou X, Ren B. Transmission routes of 2019-nCoV and controls in dental practice. Int J Oral Sci. 2020;12(1):9. 
18 - Ge ZY, Yang LM, Xia JJ, Fu XH, Zhang YZ. Possible aerosol transmission of COVID-19 and special precautions in dentistry. J Zhejiang Univ Sci B. 2020; 21(5): 361-368.

19 - Ferrazzano GF, Ingenito A, Cantile T. COVID-19 Disease in children: what dentists should know and do to prevent viral spread. the italian point of view. Int J Environ Res Public Health. 2020; 17(10): 3642.

20 - Chisini LA, Costa FS, Demarco GT, Silveira ER, Demarco FF. COVID-19 pandemic impact on paediatric dentistry treatments in the Brazilian Public Health System. Int J Paediatr Dent. 2021; 31(1): 31-34.

21 - Frencken JEFM, Flohil KA, Baat C. Atraumatic restorative treatment in relation to pain, discomfort and dental treatment anxiety. Ned Tijdschr Tandheelkd. 2014; 121(7-8): 388-93.

22 - Seangpadsa K, Smutkeeree A, Leelataweewud P. Parental acceptance of behavior management techniques for preschool children in dental practice: Revisited. J Indian Soc Pedod Prev Dent. 2020; 38(3): 274-279.

23 - Marty M, Marquet A, Valéra MC. Perception of protective stabilization by pediatric dentists: a qualitative study. JDR Clin Trans Res. 2020. [online ahead of print]. 


\title{
Management of dental caries and child' behavior during the COVID-19 pandemic: case report
}

\begin{abstract}
The aim of this article is to report a clinical case of management of dental caries and child' behavior in the scenario of the COVID-19 pandemic. One boy, seven years old, complained of caries and lack of collaboration during previous visits due to fear/anxiety. On clinical examination, cavitated caries lesions (teeth 51, 55, 61, 63, 64, 73, 74, 83, 85) and/or coronary destruction were observed (54, 75 and 84$)$ were observed. It was proposed to restore lesions without pulp involvement using the atraumatic restorative treatment technique to preserve the dental structure and reduce aerosol production, and exodontia in cases of extensive coronary destruction. To reduce child fear/ anxiety, basic techniques for managing child behavior were used. Anxiety and behavior were evaluated using scales to allow the evaluation of the efficiency of management techniques. Safety measures were explained to the child (use of facial protector, distancing in the waiting room, presence of only one companion). Selective removal of the decayed tissue and restoration with glass ionomer cement was performed in teeth $63,64,73,74,83,85$. In these procedures, the child had collaborative behavior. Teeth 75 and 84 were extracted under protective stabilization due to uncooperative behavior. At the end, the child was referred for orthodontic follow-up. There was a reduction in anxiety, improvement of behavior and control of caries disease. Minimally invasive techniques are an option for the management of dental caries. To reduce child fear/anxiety, the use of basic behavior management techniques is recommended.
\end{abstract}

KEYWORDS: Coronavirus infections; Dental caries; Child behavior.

\section{Como citar este artigo}

Barbosa MG, Silva NR, Oliveira SSB, Corrêa-Faria P. Manejo da cárie dentária e comportamento infantil durante a pandemia de COVID-19: relato de caso. Rev Odontol Bras Central 2021; 30(89): 209-221. DOI: 10.36065/robrac.v30i89.1506 